University of Wollongong

Research Online

Faculty of Engineering and Information

Faculty of Engineering and Information

Sciences - Papers: Part A

Sciences

$1-1-2015$

\title{
Phase unwrapping method based on multiple fringe patterns without use of equivalent wavelengths
}

\author{
Limei Song \\ Tianjin Polytechnic University, lilymay1976@126.com \\ Yulan Chang \\ Tianjin Polytechnic University \\ Jiangtao Xi \\ University of Wollongong, jiangtao@uow.edu.au \\ Qinghua Guo \\ University of Wollongong, qguo@uow.edu.au \\ Xin-jun Zhu \\ Tianjin Polytechnic University
}

See next page for additional authors

Follow this and additional works at: https://ro.uow.edu.au/eispapers

Part of the Engineering Commons, and the Science and Technology Studies Commons

Research Online is the open access institutional repository for the University of Wollongong. For further information contact the UOW Library: research-pubs@uow.edu.au 


\title{
Phase unwrapping method based on multiple fringe patterns without use of equivalent wavelengths
}

\begin{abstract}
In order to decrease the noise enhancement in the process of phase unwrapping, we present a new phase unwrapping approach for multiple wavelength phase shift profilometry. In this approach, a series of fringe images with only three wavelengths are captured by the 3D measurement system, and it does not need to calculate the equivalent wavelengths and the equivalent phases. As a result, the noise enhancement is avoided, leading to improved precision in the absolute phase map recovery. Experiment results demonstrate that the proposed method is more robust and effective compared to the existing TWPSP method.
\end{abstract}

\section{Keywords}

unwrapping, method, multiple, phase, fringe, wavelengths, patterns, without, equivalent

Disciplines

Engineering | Science and Technology Studies

\section{Publication Details}

L. Song, Y. Chang, J. Xi, Q. Guo, X. Zhu \& X. Li, "Phase unwrapping method based on multiple fringe patterns without use of equivalent wavelengths," Optics Communications, vol. 355, pp. 213-224, 2015.

\section{Authors}

Limei Song, Yulan Chang, Jiangtao Xi, Qinghua Guo, Xin-jun Zhu, and Xiaojie Li 


\title{
Phase unwrapping method based on multiple fringe patterns without use of equivalent wavelengths
}

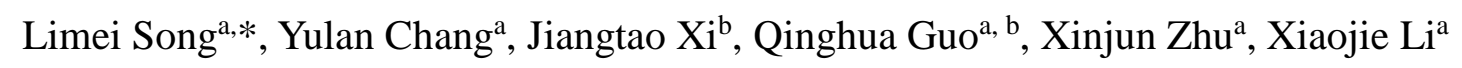

${ }^{a}$ Key Laboratory of Advanced Electrical Engineering and Energy Technology, TianJin Polytechnic University, TianJin 30087, China

${ }^{\mathrm{b}}$ School of Electrical, Computer and Telecommunications Engineering, University of Wollongong, Wollongong, NSW 2500, Australia

"lilymay1976@126.com

\begin{abstract}
In order to decrease the noise enhancement in the process of phase unwrapping, we present a new phase unwrapping approach for multiple wavelength phase shift profilometry. In this approach, a series of fringe images with only three wavelengths are captured by the 3D measurement system, and it does not need to calculate the equivalent wavelengths and the equivalent phases. As a result, the noise enhancement is avoided, leading to improved precision in the absolute phase map recovery. Experiment results demonstrate that the proposed method is more robust and effective compared to the existing TWPSP method.
\end{abstract}

\section{Keywords}

Phase unwrapping; Equivalent wavelengths; Equivalent phases; Noise enhancement

\section{Introduction}

Fringe pattern profilometry (FPP) [1] has become an effective technology for non-contact measurement of 3D shapes, which has found various applications such as 
manufacturing inspection, medical sciences, reverse engineering, quality control, etc. With FPP, Ronchi or sinusoidal grating patterns are projected on the object surface, which are deformed by the height distribution of the surface and acquired by a camera. These deformed patterns carry the 3D information of the object surface and hence can be analyzed to extract the information. In order to reconstruct the 3D shape, various fringe pattern analysis methods have been developed, e.g., Fourier transform profilometry (FTP) [2], phase shift profilometry (PSP) [3], modulation measurement profilometry (MMP) [4], phase-measuring profilometry (PMP) [5], etc.

Among these approaches, PSP is particularly commonly used due to the advantages such as high speed, high resolution, high accuracy and its non-contact nature. Moreover, the PSP is less sensitive to the surface reflectivity variations and the object can be measured on pixel-by-pixel basis. With PSP, multiple fringe stripes [6] with the same wavelength are usually utilized. However, the phase maps retrieved are wrapped ones, which must be unwrapped for 3D shape recovery. In order to unwrap the phase maps, many different algorithms are developed, e.g., spatial phase unwrapping [7] and temporal phase unwrapping methods [8]. Spatial phase unwrapping is based on the analysis of adjacent or neighboring pixels on an individual wrapped phase map, while the temporal phase unwrapping is a type of method based on analysis of multiple pattern images projected sequentially [9]. In contrast to the spatial approaches, the temporal ones are believed to be more robust, but they are less efficient in terms of speed due to the use of multiple pattern images.

The temporal phase unwrapping techniques can be classified as the Coding PSP method and the Multi Wavelength PSP (MWPSP) method. The coding PSP method tries to achieve phase unwrapping by associating the fringe strips with a digital codeword, which is usually implemented by projection of additional pattern images 
such as Gray-code [10-11]. While the coding PSP is able to achieve robust phase unwrapping, they require projection of additional encoded pattern images.

The MWPSP method is based on projection of multiple fringe patterns with different wavelengths. In MWPSP method, a series of phase shift fringe images with different wavelengths are captured. Phase unwrapping is carried out, on one-by-one basis, from the phase map with the longest wavelength to the one with the shortest, where the unwrapped phase map is employed as the reference to recover the next phase map of the next shorter wavelength. Chen, et al. [12] described a novel technique to handle the $2 \pi$ phase ambiguity problem through utilizing two properly chosen frequency gratings to synthesize an equivalent wavelength grating. Towers, et al. [13-14] proposed an optimal frequency selection for the multiple wavelength method. Later they verified, by simulation, an optimal three wavelength for 3D shape measurement. $\mathrm{Li}$, et al. [15] presented a phase unwrapping approach based on multiple wavelength scanning for digital holographic microscopy, which unwrapped the ambiguous phase image layer by layer by synthesizing the extracted continuous components from a set of multiple phase images obtained by varying the optical wavelength. Ding, et al. [16-17] proposed an approach to recover absolute phase maps of two fringe patterns with selected frequencies, and later they provided a guideline for frequency selection. Long, et al. [18] proposed a method which can be applied for any two fringe patterns with different fringe wavelengths through choosing the fringe waveform lengths instead of using the two frequencies to describe the two fringe patterns.

Due to the existence of noise in the environment when we project the fringe patterns, multiple intermediate fringe patterns must be used, and so the result of the unwrapping will have error. In order to remedy the problem in PSP phase unwrapping 
described above, we already developed a phase unwrapping algorithm based on three wavelength phase shift profilometry (TWPSP) method [19]. However, the proposed technique in [19] still needs to calculate the equivalent phases through the equivalent wavelengths. The noise associated with the wrapped phase map will propagate to the unwrapped ones, leading to errors in the recovered phase maps.

In this paper, we present a new phase unwrapping approach based on the use of three fringe patterns with different wavelengths. Compared with the existing method in [19-20], the proposed method does not need to calculate the equivalent phases. As the noise propagation and enhancement are avoided, the resulting unwrapped phase is less influenced by the noise, therefore leading to improved phase unwrapping performance.

This paper is organized as follows. Section 2 presents the principle of the TWPSP method and the proposed TWPSP approach. Section 3 shows simulation and experimental results and the comparisons with the existing 3D reconstruction methods. Section 4 discusses the advantages of the proposed method compared with the existing TWPSP method, the GCPSP method, and the MWPSP method.

\section{The principle of the Three Wavelength PSP (TWPSP) method}

\subsection{The principle of the existing TWPSP method}

Taking the three classical wavelengths $\lambda_{1}=21, \lambda_{2}=18$ and $\lambda_{3}=16$ as an example to explain the TWPSP method which uses three short wavelengths to generate an equivalent long wavelength. Assuming that the three short wavelengths are $\lambda_{1}, \lambda_{2}$ and $\lambda_{3}$, and the corresponding wrapped phase maps are $\phi_{1}(x, y), \phi_{2}(x, y)$ and $\phi_{3}(x, y)$, phase maps $\phi_{12}(x, y), \quad \phi_{23}(x, y)$ and $\phi_{123}(x, y)$ can be obtained with their equivalent wavelengths $\lambda_{12}, \lambda_{23}$ and $\lambda_{123}$ being calculated by: 


$$
\lambda_{12}=\left|\frac{\lambda_{1} \times \lambda_{2}}{\lambda_{1}-\lambda_{2}}\right|, \lambda_{23}=\left|\frac{\lambda_{2} \times \lambda_{3}}{\lambda_{2}-\lambda_{3}}\right|, \lambda_{123}=\left|\frac{\lambda_{12} \times \lambda_{23}}{\lambda_{12}-\lambda_{23}}\right| .
$$

For the three classical wavelength values $\lambda_{1}=21, \lambda_{2}=18$ and $\lambda_{3}=16$, we can get $\lambda_{12}=126, \lambda_{23}=144$ and $\lambda_{123}=1008$. For the four steps PSP algorithm, four pattern images are projected, and the captured pattern can be represented in the following:

$$
I_{k}(x, y)=a(x, y)+b(x, y) \sin \left(\phi(x, y)+\frac{\pi \times k}{2}\right), k=0,1,2,3
$$

where $a(x, y)$ is the background intensity, $b(x, y)$ is the intensity modulation, $\phi(x, y)$ is the wrapped phase.

We have showed in [19] that the wrapped phase value $\phi_{i}(x, y)$ can be calculated as:

$$
\phi_{i}(x, y)=\arctan \left(\frac{I_{0}(x, y)-I_{2}(x, y)}{I_{1}(x, y)-I_{3}(x, y)}\right), i=1,2,3 .
$$

From Eq. (1) and Eq. (3), the phase maps $\phi_{12}(x, y), \phi_{23}(x, y)$ and $\phi_{123}(x, y)$ can be obtained by:

$$
\begin{aligned}
& \phi_{12}(x, y)=\arctan \left(\frac{\sin \left(\phi_{2}(x, y)\right) \times \cos \left(\phi_{1}(x, y)\right)-\cos \left(\phi_{2}(x, y)\right) \times \sin \left(\phi_{1}(x, y)\right)}{\cos \left(\phi_{2}(x, y)\right) \times \cos \left(\phi_{1}(x, y)\right)+\sin \left(\phi_{2}(x, y)\right) \times \sin \left(\phi_{1}(x, y)\right)}\right) \\
& \phi_{23}(x, y)=\arctan \left(\frac{\sin \left(\phi_{3}(x, y)\right) \times \cos \left(\phi_{2}(x, y)\right)-\cos \left(\phi_{3}(x, y)\right) \times \sin \left(\phi_{2}(x, y)\right)}{\cos \left(\phi_{3}(x, y)\right) \times \cos \left(\phi_{2}(x, y)\right)+\sin \left(\phi_{3}(x, y)\right) \times \sin \left(\phi_{2}(x, y)\right)}\right) \\
& \phi_{123}(x, y)=\arctan \left(\frac{\sin \left(\phi_{12}(x, y)\right) \times \cos \left(\phi_{23}(x, y)\right)-\cos \left(\phi_{12}(x, y)\right) \times \sin \left(\phi_{23}(x, y)\right)}{\cos \left(\phi_{12}(x, y)\right) \times \cos \left(\phi_{23}(x, y)\right)+\sin \left(\phi_{12}(x, y)\right) \times \sin \left(\phi_{23}(x, y)\right)}\right)
\end{aligned}
$$

We assume that there are $\mathrm{W}$ pixels along the direction vertical to the fringe strips. If the equivalent wavelength $\lambda_{123}$ meets the condition $\lambda_{123} \geq \mathrm{W}, \phi_{123}(x, y)$ varies from 0 to $2 \pi$ without $2 \pi$ discontinuities, and then the figures of the wrapped phase can be clearly expression. In this case, phase unwrapping is not required, because the absolute phase map is the same as the wrapped one, i.e., $\Phi_{123}(x, y) \equiv \phi_{123}(x, y)$. 
Although $\phi_{123}(x, y)$ does not exhibit $2 \pi$ discontinuities, reconstruction of the $3 \mathrm{D}$ surface directly from $\phi_{123}(x, y)$ will not achieve good results, because long wavelength usually results in low measurement accuracy. If the $\phi_{1}(x, y)$ can be unwrapped according to the equivalent phases, the result will be better. However, on $\phi_{1}(x, y)$, there are $\lambda_{12} / \lambda_{1}$ discontinuities with equal spacing $\lambda_{12}$, and within each $\lambda_{12}$ there are $\lambda_{123} / \lambda_{12}$ discontinuities on $\phi_{12}(x, y)$. Hence, there are altogether $\frac{\lambda_{123}}{\lambda_{12}} \times \frac{\lambda_{12}}{\lambda_{1}}$ discontinuities on $\phi_{1}(x, y)$.

The absolute phase $\Phi_{1}(x, y)$ with wavelength $\lambda_{1}$ can be calculated as:

$$
\begin{aligned}
& \Phi_{1}(x, y)=\phi_{1}(x, y)+2 \pi\left(\operatorname{int}\left(\frac{\phi_{123}(x, y)}{\frac{2 \pi}{\lambda_{123}}}\right) \times \frac{\lambda_{12}}{\lambda_{1}}+\operatorname{int}\left(\frac{\phi_{12}(x, y)}{\frac{2 \pi}{\lambda_{12}}}\right)\right) \\
& =\phi_{1}(x, y)+2 \pi\left(\operatorname{int}\left(\frac{\phi_{123}(x, y)}{2 \pi} \times \frac{\lambda_{123}}{\lambda_{12}}\right) \times \frac{\lambda_{12}}{\lambda_{1}}+\operatorname{int}\left(\frac{\phi_{12}(x, y)}{2 \pi} \times \frac{\lambda_{12}}{\lambda_{1}}\right)\right)
\end{aligned}
$$

where int (a) returns an integer close to a.

In the above, we did not consider the impact of measurement noise. Due to the existence of the measurement noise, the four pattern images can be represented as:

$$
I_{k}(x, y)=a(x, y)+b(x, y) \sin \left(\phi(x, y)+\frac{\pi \times k}{2}\right)+\operatorname{Gau}(x, y), k=0,1,2,3
$$

where $\operatorname{Gau}(x, y)$ denotes white Gaussian noise. The measurement noise will be propagated and even enhanced in the calculations of the equivalent phases with Eq. (4), thereby leading to performance degradation in the calculations of the absolute phased with (5).

\subsection{The principle of the proposed TWPSP method}

A new method is proposed in this section to avoid the above mentioned problem. We 
still assume that there are $W$ pixels in the direction vertical to the fringe strips, and three fringe patterns are projected with wavelengths $\lambda_{1}{ }^{\prime}=1008, \lambda_{2}{ }^{\prime}=144$ and $\lambda_{3}{ }^{\prime}=16$ respectively, and we assume $\lambda_{1}{ }^{\prime} \geq W$. We still simulate four pattern images with Eq. (6), and add the same noise to the signal. For every wavelength, the corresponding wrapped phase value phi_1 $\phi_{1}{ }^{\prime}(x, y)$, phi_2 $\phi_{2}{ }^{\prime}(x, y)$ and phi_3 $\phi_{3}{ }^{\prime}(x, y)$ can be calculated directly by Eq. (3), and Eq. (4) is not needed. In order to achieve this, the three wavelengths are chosen to meet the following relationships:

$$
N_{1}=\lambda_{1}{ }^{\prime} / \lambda_{2}{ }^{\prime}, N_{2}=\lambda_{2}{ }^{\prime} / \lambda_{3}{ }^{\prime}
$$

where $N_{1}$ and $N_{2}$ must be integers. As phi_1 does not have any discontinuity, there are maximally $N_{1}$ discontinuities on phi_2. Also, for each $\lambda_{2}{ }^{\prime}$ pixels on phi_3, there are $N_{2}$ discontinuities. Therefore, in total there are maximally $N_{1} \times N_{2}$ discontinuities on phi_3, which can be eliminated by phase unwrapping operation below, yielding the absolute phase $\Phi_{3}(x, y)$ as follows:

$$
\begin{aligned}
& \Phi_{3}(x, y)=\phi_{3}{ }^{\prime}(x, y)+2 \pi\left(\operatorname{int}\left(\frac{\phi_{1}{ }^{\prime}(x, y)}{\frac{2 \pi}{N_{1}}}\right) \times N_{2}+\operatorname{int}\left(\frac{\phi_{2}{ }^{\prime}(x, y)}{\frac{2 \pi}{N_{2}}}\right)\right) \\
& =\phi_{3}{ }^{\prime}(x, y)+2 \pi\left(\operatorname{int}\left(\frac{\phi_{1}{ }^{\prime}(x, y)}{2 \pi} \times N_{1}\right) \times N_{2}+\operatorname{int}\left(\frac{\phi_{2}{ }^{\prime}(x, y)}{2 \pi} \times N_{2}\right)\right)
\end{aligned}
$$

Note that when $\phi_{3}{ }^{\prime}(x, y)=2 \pi$, a discontinuity will occur. In order to remove such a discontinuity, the phase unwrapping equation of $\Phi_{3}(x, y)$ should be revised to:

$$
\Phi_{3}(x, y)=\left\{\begin{array}{l}
\phi_{3}^{\prime}(x, y)+2 \pi\left(\operatorname{int}\left(\frac{\phi_{1}(x, y)}{2 \pi} \times N_{1}\right) \times N_{2}+\operatorname{int}\left(\frac{\phi_{2}^{\prime}(x, y)}{2 \pi} \times N_{2}\right)\right), \quad \phi_{3}^{\prime}(x, y) \neq 2 \pi \text { and } \phi_{2}{ }^{\prime}(x, y) \neq 2 \pi \text { and } \phi_{1}{ }^{\prime}(x, y) \neq 2 \pi \\
\phi_{3}^{\prime}(x, y)+2 \pi\left(\operatorname{int}\left(\frac{\phi_{1}^{\prime}(x, y)}{2 \pi} \times N_{1}\right) \times N_{2}+\operatorname{int}\left(\frac{\phi_{2}^{\prime}(x, y)}{2 \pi} \times N_{2}\right)-1\right), \quad\left(\phi_{3}^{\prime}(x, y)=2 \pi \text { or } \phi_{2}^{\prime}(x, y)=2 \pi\right) \text { and } \phi_{1}^{\prime}(x, y) \neq 2 \pi \\
\phi_{3}^{\prime}(x, y)+2 \pi\left(\operatorname{int}\left(\frac{\phi_{1}^{\prime}(x, y)}{2 \pi} \times N_{1}-1\right) \times N_{2}+\operatorname{int}\left(\frac{\phi_{2}^{\prime}(x, y)}{2 \pi} \times N_{2}\right)\right), \quad \phi_{3}^{\prime}(x, y) \neq 2 \pi \text { and } \phi_{2}^{\prime}(x, y) \neq 2 \pi \text { and } \phi_{1}^{\prime}(x, y)=2 \pi
\end{array}\right.
$$


Hence the formula for unwrapping the phase map phi_3 is obtained. Although Eq. (8) is similar to Eq. (5), the wrapped phase $\phi_{1}{ }^{\prime}(x, y), \phi_{2}{ }^{\prime}(x, y)$ can be directly calculated by Eq. (3), that is, the calculations of the equivalent wavelengths and their phases are not required, thereby improving the performance of absolute phase recovery, and the phase unwrapping is more robust.

\section{Simulation and Experiment}

In order to demonstrate the advantages of the proposed method, simulations and experiments have been carried out in our laboratory, where the fringe patterns covers 800 pixels along the direction vertical to the fringe strips, that is $\mathrm{W}=800$. For the existing TWPSP method, the wavelengths are set as $\lambda_{1}=21, \lambda_{2}=18$ and $\lambda_{3}=16$. For the proposed method, the three wavelengths are set to $\lambda_{1}{ }^{\prime}=1008, \lambda_{2}{ }^{\prime}=144$ and $\lambda_{3}{ }^{\prime}=16$.

In the simulations, we add the white Gaussian noises with three different SNRs (signal to noise ratio) to the four pattern images. The recovered wrapped phases of three wavelengths $\lambda=1008, \lambda=144$ and $\lambda=16$ are shown in Figs. 1-3 (with different SNRs: SNR=10dB in Fig. 1, SNR=15dB in Fig. 2 and SNR=20dB in Fig. 3.), where the wrapped phases by the existing TWPSP method are also shown for comparisons .

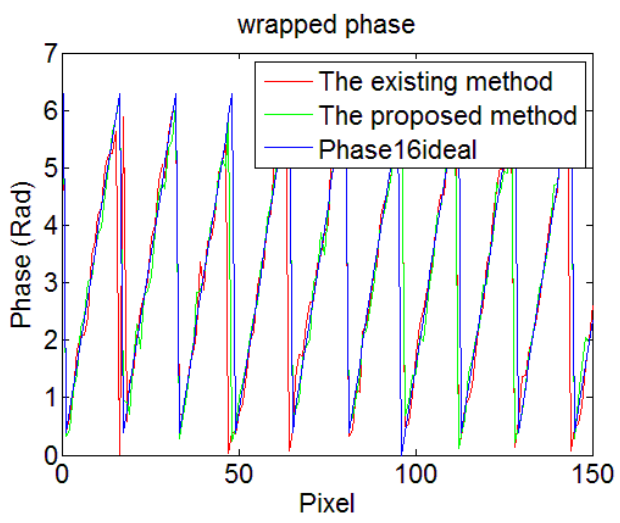

(a)

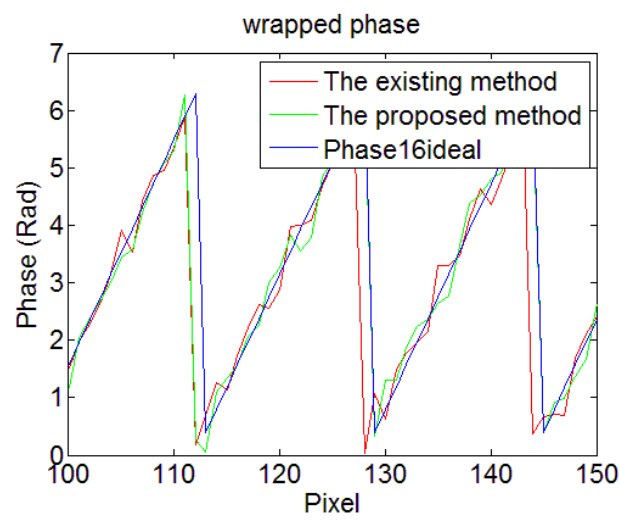

(b) 


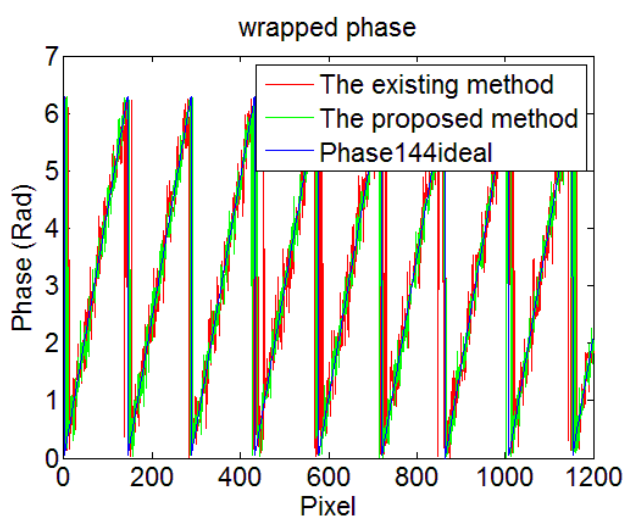

(c)

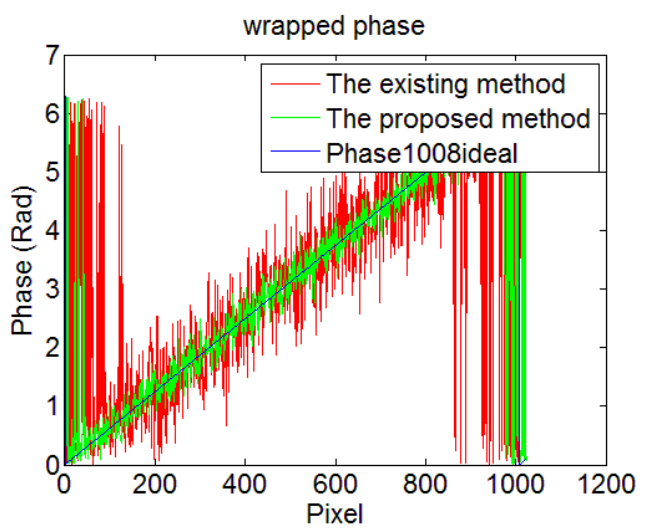

(e)

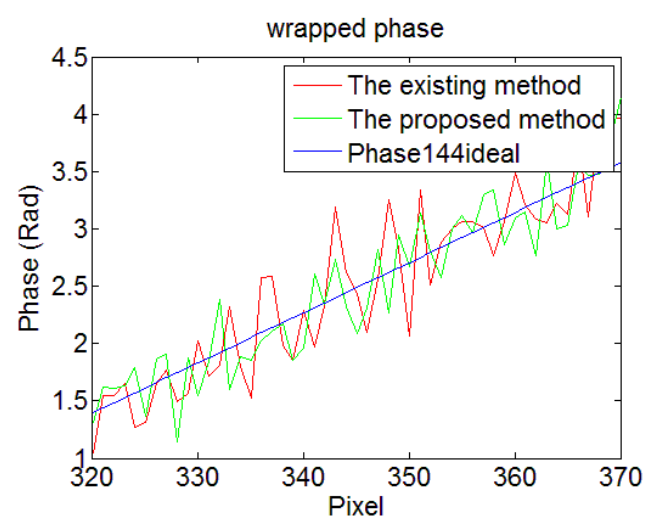

(d)

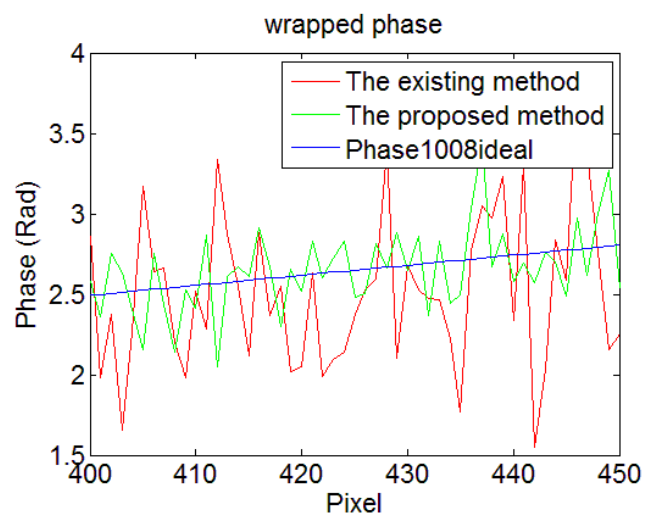

(f)

Fig. 1. The wrapped phase with the three wavelengths when $\mathrm{SNR}=10 \mathrm{~dB}$. (a)The phases with $\lambda=16$ for the existing TWPSP method, the proposed TWPSP method and the ideal TWPSP method (assuming no noise). (b)The zoomed view of Fig. 1(a). (c)The phases with $\lambda=144$ for the existing TWPSP method, the proposed TWPSP method and the ideal TWPSP method. (d) The zoomed view of Fig. 1(c). (e)The phases with $\lambda=1008$ for the existing TWPSP method, the proposed TWPSP method and the ideal TWPSP method. (f)The zoomed view of Fig. 1(e). 


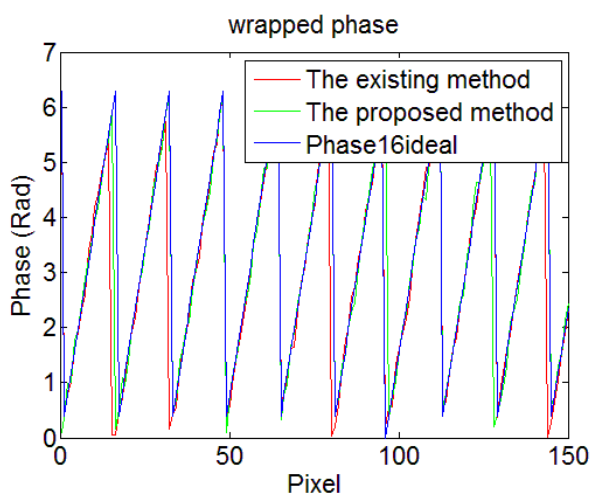

(a)

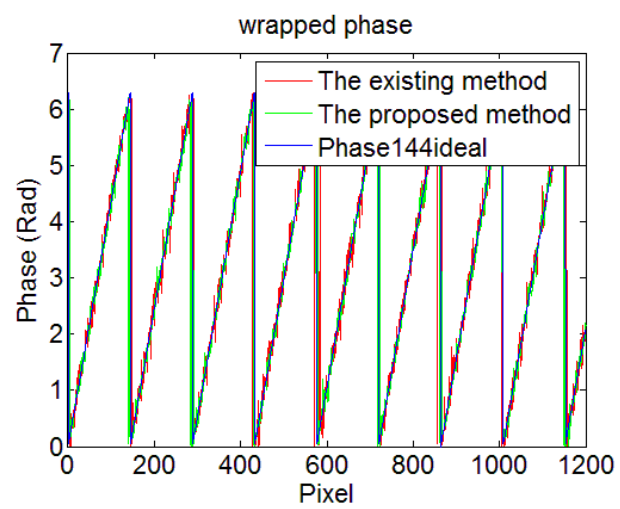

(c)

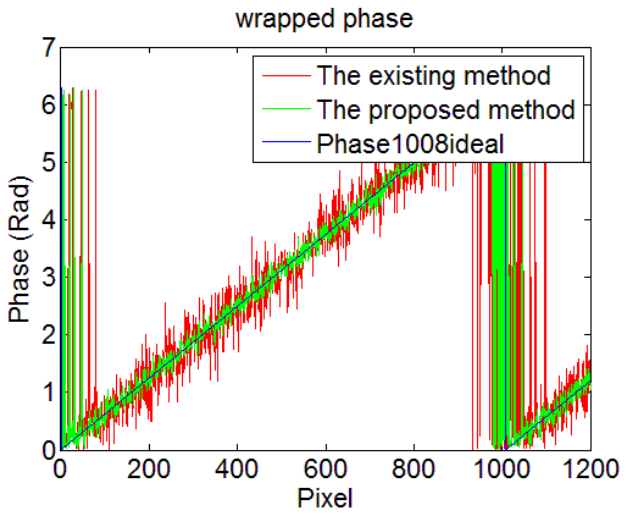

(e)

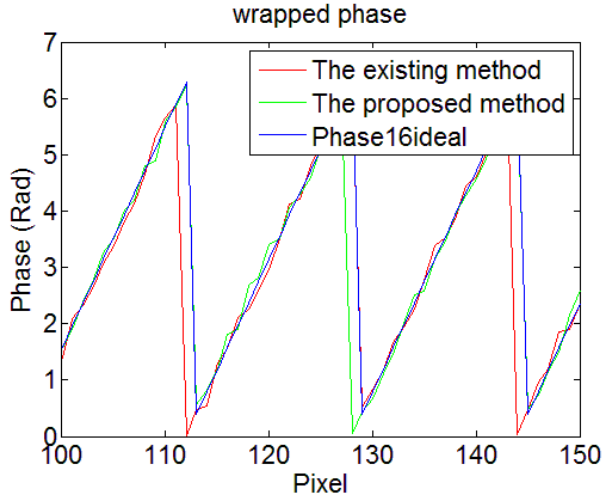

(b)

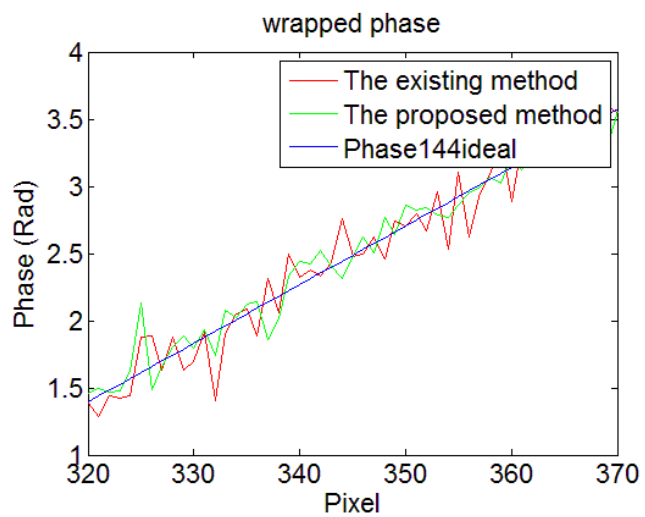

(d)

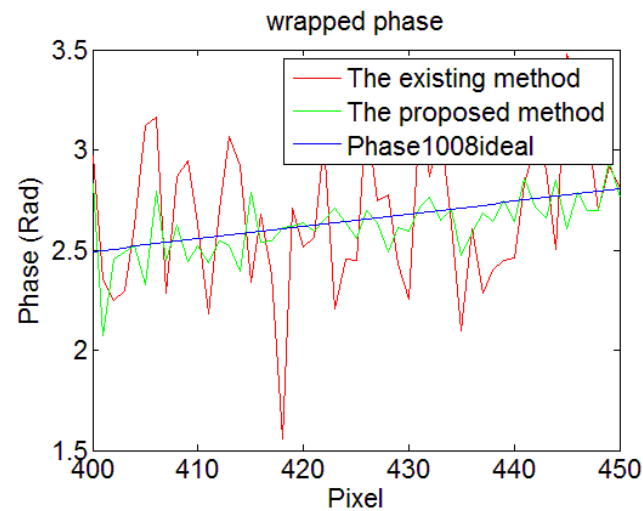

(f)

Fig. 2. The wrapped phase with the three wavelengths when $\mathrm{SNR}=15 \mathrm{~dB}$. (a)The phases with $\lambda=16$ for the existing TWPSP method, the proposed TWPSP method and the ideal TWPSP method (assuming no noise). (b)The zoomed view of Fig. 2(a). (c)The phases with $\lambda=144$ for the existing TWPSP method, the proposed TWPSP method and the ideal TWPSP method. (d) The zoomed view of Fig. 
2(c). (e)The phases with $\lambda=1008$ for the existing TWPSP method,

the proposed TWPSP method and the ideal TWPSP method. (f)The zoomed view of Fig. 2(e).

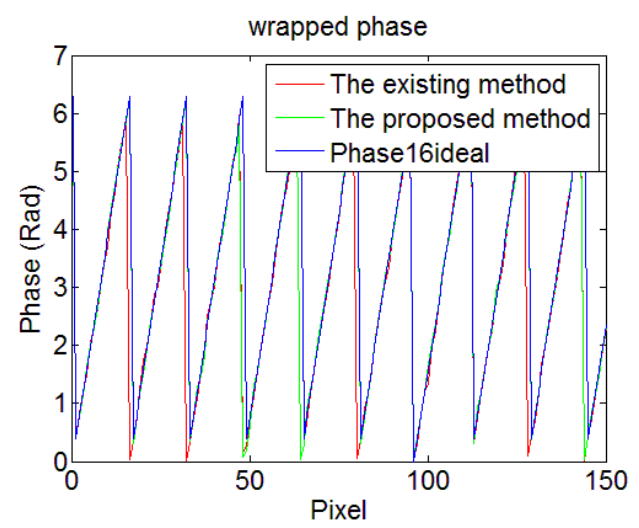

(a)

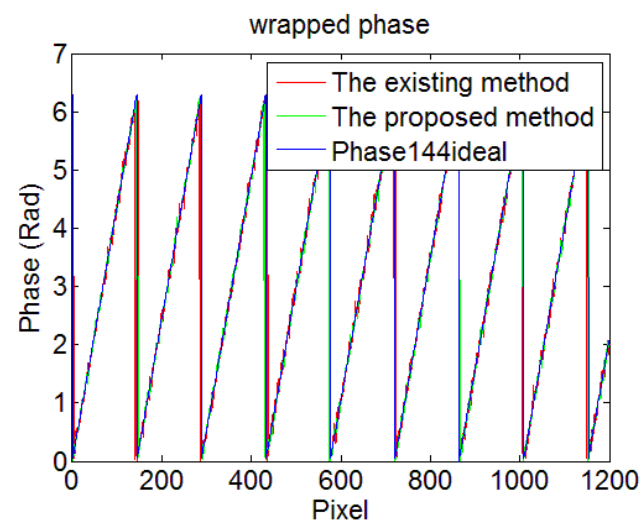

(c)

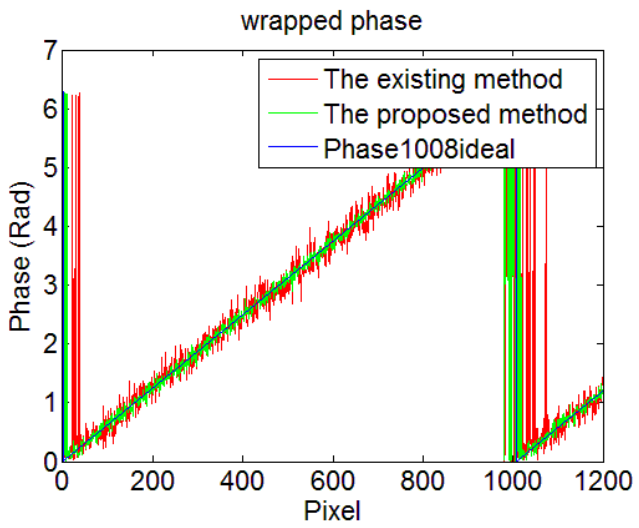

(e)

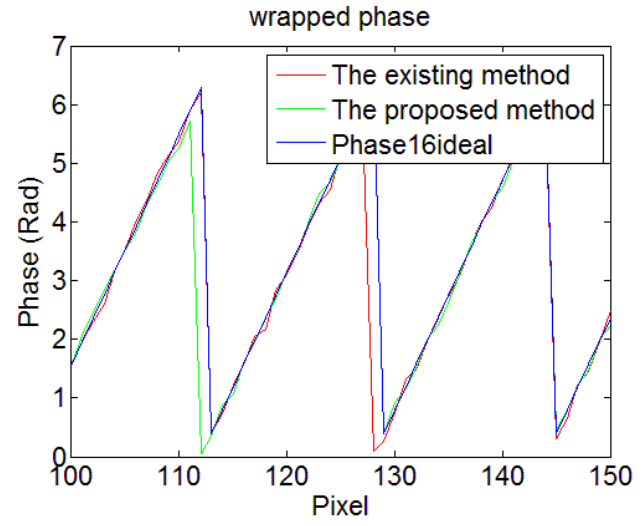

(b)

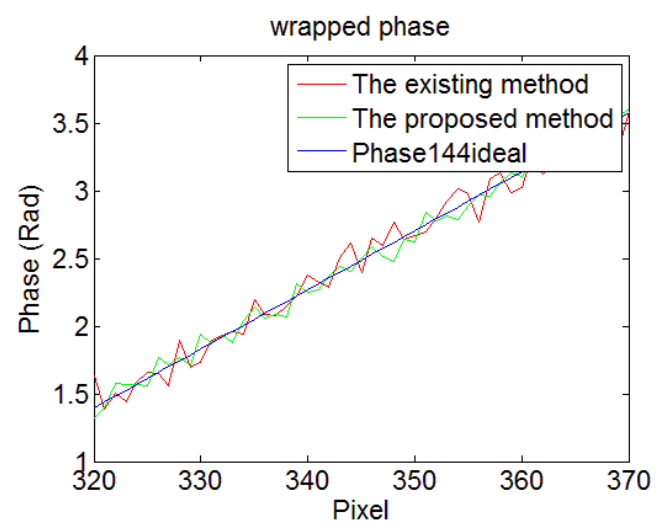

(d)

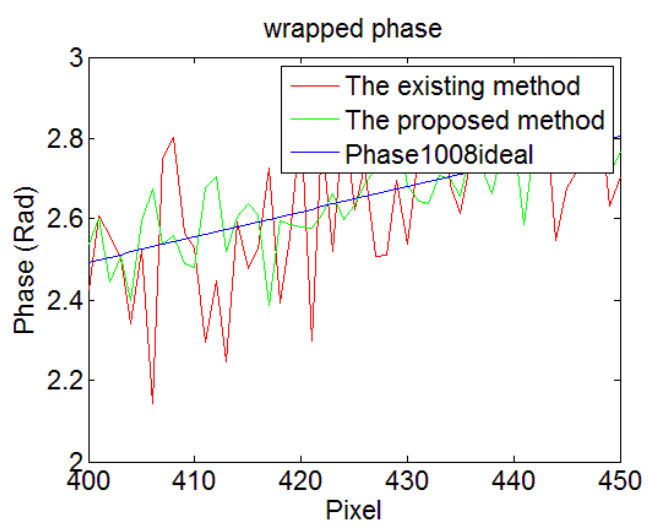

(f)

Fig. 3. The wrapped phase with the three wavelengths when $\mathrm{SNR}=20 \mathrm{~dB}$. (a)The phases with $\lambda=16$ for the existing TWPSP method, the proposed TWPSP method and the ideal TWPSP method 
(assuming no noise). (b)The zoomed view of Fig. 3(a). (c)The phases with $\lambda=144$ for the existing TWPSP method, the proposed TWPSP method and the ideal TWPSP method. (d) The zoomed view of Fig. 3(c). (e)The phases with $\lambda=1008$ for the existing TWPSP method, the proposed TWPSP method and the ideal TWPSP method. (f)The zoomed view of Fig. 3(e).

The red lines of Fig. i(a) $(i=1,2,3)$ denote the wrapped phase, for the existing TWPSP method with $\lambda_{3}$, and the green lines of Fig. i(a) $(i=1,2,3)$ represent the wrapped phase for the proposed TWPSP method with $\lambda_{3}{ }^{\prime}$. Because $\lambda_{3}=\lambda_{3}{ }^{\prime}$ in the two methods, and the equations for calculating their wrapped phases are the same, their wrapped phases are almost the same. The wrapped phase phi_12 is computed from patterns 1 and 2, and it is shown by the red lines of Fig. $i(c)(i=1,2,3)$; while the wrapped phase of $\lambda_{2}{ }^{\prime}$ for the proposed TWPSP method is shown by the green lines of Fig. i(c) (i=1,2,3). The wrapped phase phi_123 for the existing TWPSP method is shown by the red lines of Fig. i(e) $(i=1,2,3)$; while the wrapped phase phi_1 for the proposed method is shown by the green lines of Fig. $\mathrm{i}(\mathrm{e})(\mathrm{i}=1,2,3)$, and the blue lines of Fig. i represent the ideal wrapped phases. To comparisons, parts of Fig. i(a), Fig. i(c), Fig. i(e) $(i=1,2,3)$ have been zoomed in, and are shown in Fig. i(b), Fig. i(d), Fig. $\mathrm{i}(\mathrm{f})(\mathrm{i}=1,2,3)$, respectively.

From the simulations results shown in Figs. 1-3, the error propagation and noise enhancement in the existing TWSP method can be clearly observed (by comparing the red curves in the Fig. i(b) Fig. i(d), Fig. i(f) $(i=1,2,3)$. We can also see that the proposed method achieves significant performance improvement thanks to the avoidance of the calculation of the equivalent phase maps. Next, we analyze the impact of the noise quantitatively. For this, 200 points in the phase map (from 400 
to599) are selected, and the root mean squared errors (RMSE) are compared and shown in Table 1, where the RMSE is defined as:

$$
S=\sqrt{\left[\left(x_{1}-x_{\text {ideal }, 1}\right)^{2}+\left(x_{2}-x_{\text {ideal }, 2}\right)^{2}+\cdots+\left(x_{n}-x_{\text {ideal }, n}\right)^{2}\right] / n}
$$

where $\mathrm{S}$ is the RMSE, $x_{\text {ideal }, n}$ is the ideal wrapped phase of the point $\mathrm{n}, x_{n}$ is the wrapped phase of the point $n, n$ is the point number.

Table 1: The root mean square errors (RMSE) of the two methods

\begin{tabular}{|c|c|c|c|}
\hline$X$ & $\begin{array}{l}\text { The ideal } \\
\text { wrapped phase } \\
\text { of } \lambda=1008 \\
\text { (Rad) }\end{array}$ & $\begin{array}{l}\text { The wrapped } \\
\text { phase of } \lambda_{1}{ }^{\prime} \text { in the } \\
\text { proposed method } \\
\text { (Rad) }\end{array}$ & $\begin{array}{l}\text { The wrapped } \\
\text { phase of } \lambda_{123} \text { in the } \\
\text { existing method } \\
\text { (Rad) }\end{array}$ \\
\hline $\begin{array}{c}\text { RMSE with } \\
\text { SNR }=10 \mathrm{~dB}\end{array}$ & 0 & 0.2421 & 0.5626 \\
\hline $\begin{array}{c}\text { RMSE with } \\
\text { SNR }=15 \mathrm{~dB}\end{array}$ & 0 & 0.1246 & 0.3169 \\
\hline $\begin{array}{c}\text { RMSE with } \\
\text { SNR }=20 \mathrm{~dB}\end{array}$ & 0 & 0.0642 & 0.1760 \\
\hline
\end{tabular}

From the above table, it can be seen that these RMSEs of the proposed method are smaller than those of the existing method, and the difference becomes larger with the decrease of the SNR, i.e., compared to the existing method, the proposed method is more prominent when the measurement noise is larger.

Next, experimental results are provided to verify the proposed method. In the experiment, the fringe patterns are projected onto a plaster hand model, and the captured images are shown in Fig. 4. 


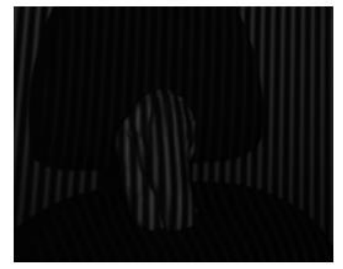

$\lambda_{1}=21$

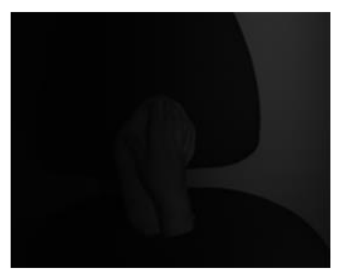

$\lambda_{1}^{\prime}=1008$

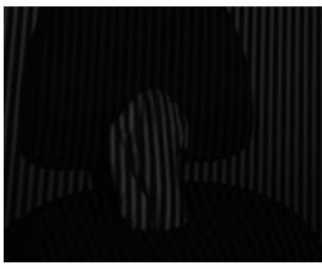

$\lambda_{2}=18$

(a)

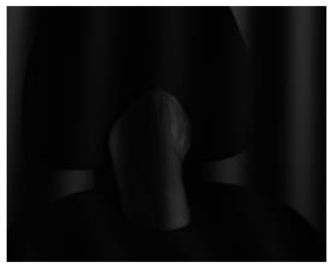

$\lambda_{2}^{\prime}=144$

(b)

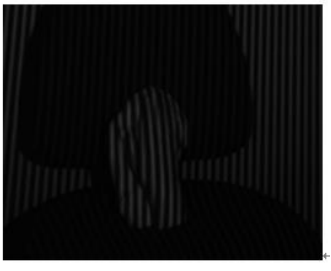

$\lambda_{3}=16$

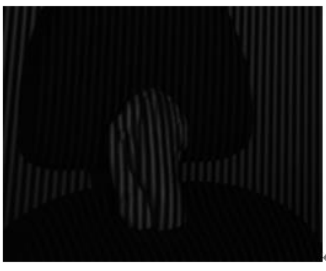

$\lambda_{3}^{\prime}=16$

Fig. 4. Projecting fringe patterns with different wavelengths. (a)The existing TWPSP method. (b) The proposed TWPSP method.

For the existing TWPSP method in [19], the three original wavelengths are used to calculate the equivalent wavelength $\lambda_{12}, \lambda_{23}$ and $\lambda_{123}$ based on Eq. (1), yielding $\lambda_{12}=126, \lambda_{23}=144$ and $\lambda_{123}=1008$. The wrapped phase maps from the three original and equivalent wavelength of the existing TWPSP method are shown in Fig.5(a). For the proposed TWPSP method, the wavelength $\lambda_{1}{ }^{\prime}$ in the proposed method is equal to the equivalent wavelength $\lambda_{123}$ used in the existing TWPSP method. The wavelength $\lambda_{2}{ }^{\prime}$ in the proposed method is equal to the equivalent wavelength $\lambda_{23}$ used in the existing TWPSP method. Only $\lambda_{3}{ }^{\prime}$ is the same for the two methods. The wrapped phase maps from the proposed TWPSP method are shown in Fig. 5(b). 

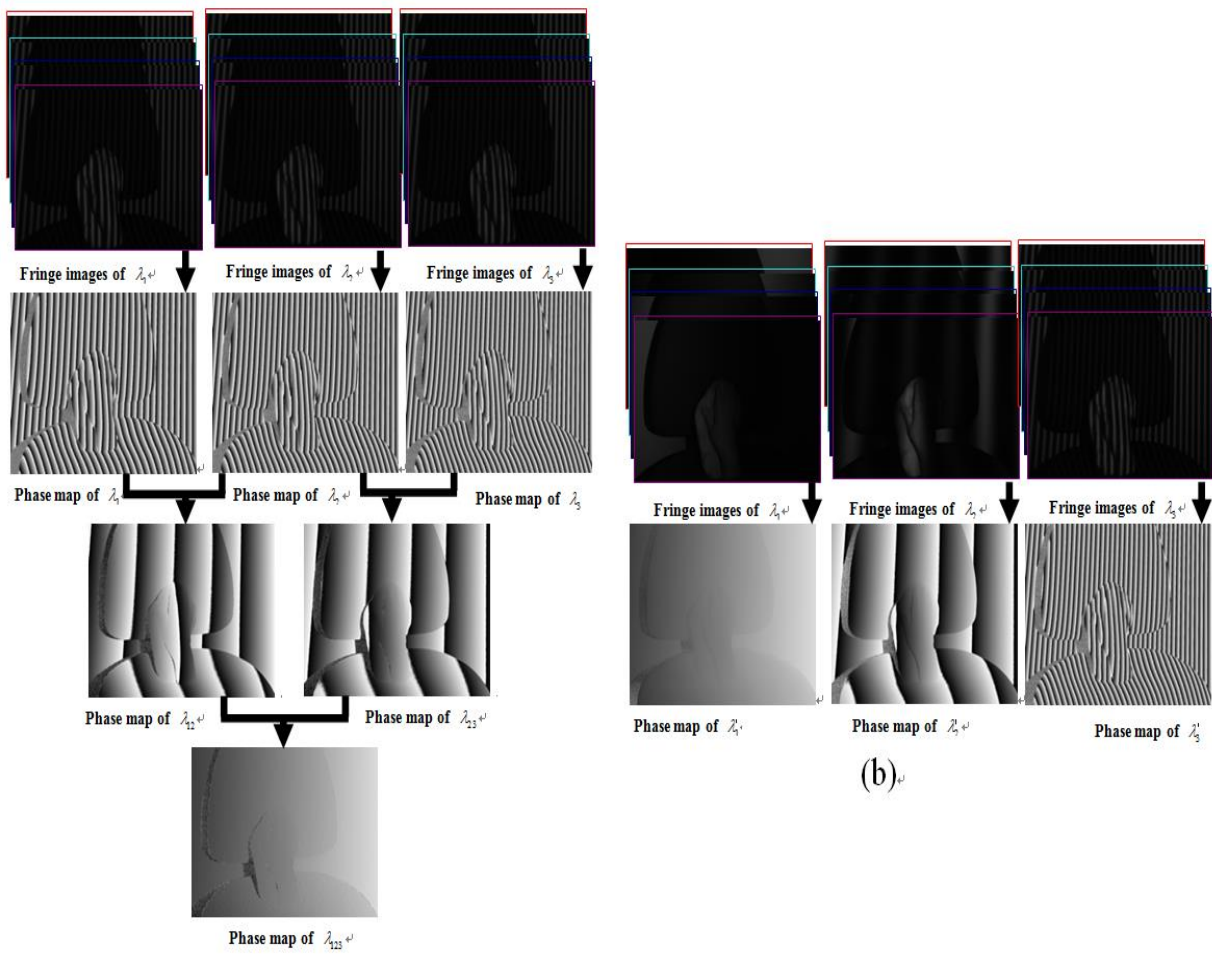

(b)

(a).

Fig. 5. The acquisition of the wrapped phase from the two methods. (a)

The acquisition of the wrapped phase from the three original and equivalent wavelength of the existing TWPSP method. (b) The acquisition of the wrapped phases from the proposed TWPSP method

From the comparison of Fig.5(a) and Fig.5(b), the existing TWPSP method needs to calculate twice equivalent wavelengths, while the equivalent wavelengths are not required to be calculated in the proposed method. So the existing TWPSP is more complex and time consuming than the proposed method.

Now let us consider a row of pixels in the fringe images on the row 512. In the experiments, Fig. 6(a) shows the wrapped phase of image 3 (with wavelength $\lambda_{3}$ ) from the existing TWPSP method, and Fig. 6(b) gives the wrapped phase of $\lambda_{3}{ }^{\prime}$ from the proposed TWPSP method. Because the two pattern images come from the same object and the wavelength is same, they are almost the same. The wrapped phase phi_23 is computed from patterns 1 and 2, and it is shown in Fig. 7(a); while 
the wrapped phase of $\lambda_{2}$ from the proposed TWPSP method is shown in Fig. 7(b).

The wrapped phase phi_123 from the existing TWPSP method is shown in Fig. 8(a); while the wrapped phase phi_1 from the proposed method is shown in Fig. 8(b).

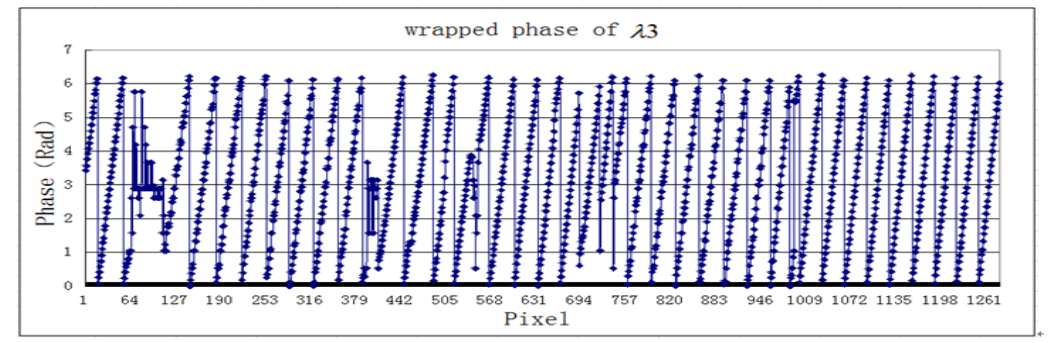

(a)

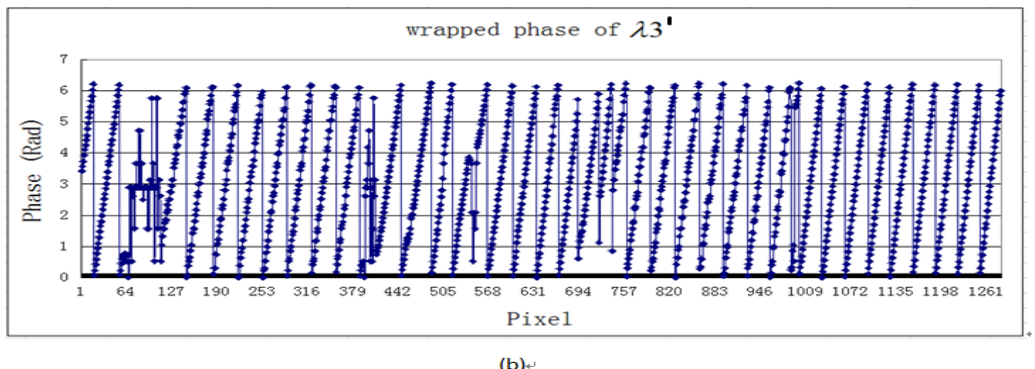

Fig. 6. (a)The wrapped phase of $\lambda_{3}$ from the existing TWPSP method (b)The wrapped phase of $\lambda_{3}{ }^{\prime}$ from the proposed TWPSP method.

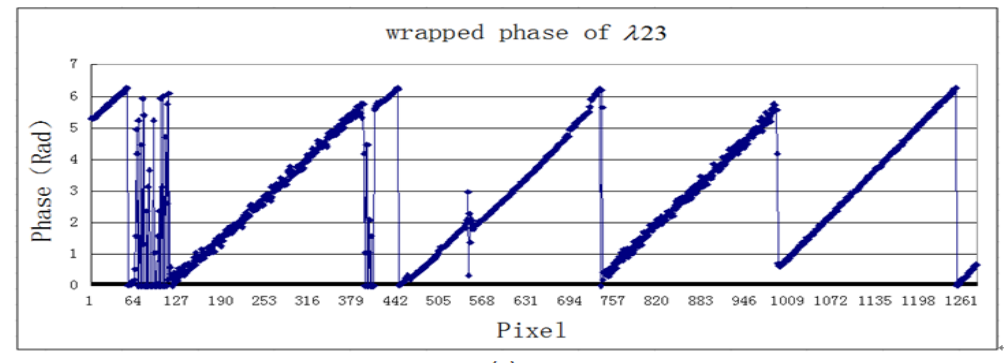

(a)

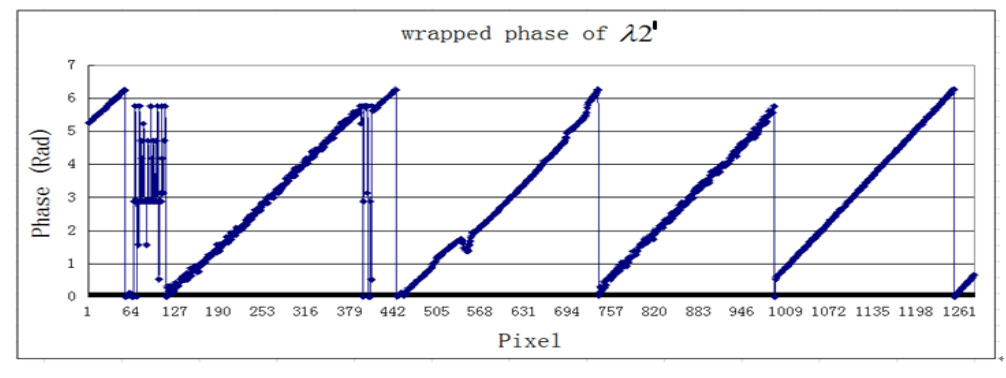

(b)

Fig. 7. (a) The wrapped phase of $\lambda_{23}$ from the existing TWPSP method.

(b) The wrapped phase of $\lambda_{2}$ ' from the proposed TWPSP method. 


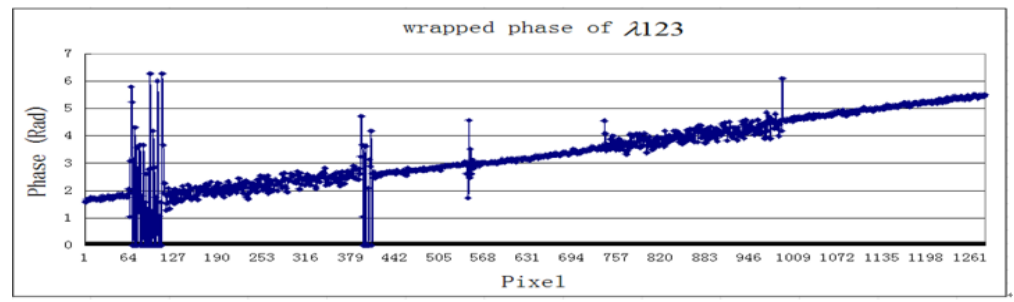

(a).

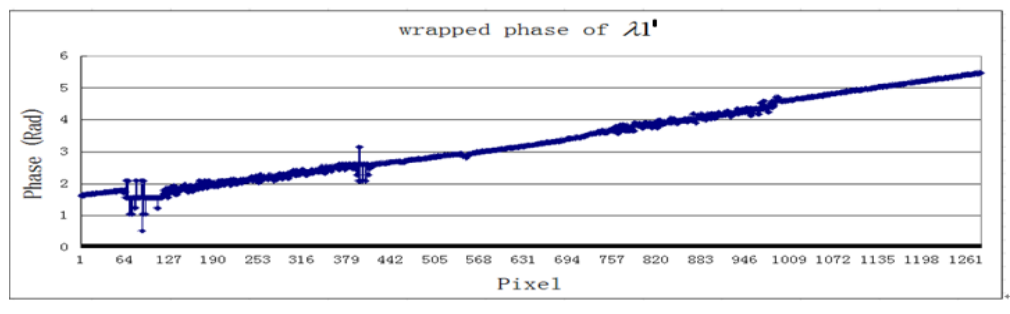

(b).

Fig. 8. (a)The wrapped phase of $\lambda_{123}$ from the existing TWPSP method. (b) The wrapped phase of $\lambda_{1}{ }^{\prime}$ from the proposed TWPSP method

In the experiment, phi_23 is calculated from phi_2 and phi_3, so the noise on the latter will be transferred to the former. By comparing Fig. 7(a) and Fig. 7(b), it is seen that phi_2 from the proposed method is better than phi_23. From the comparison of Fig. 8(a) and Fig. 8(b), phi_1 from the proposed method is much better than the phi_123 from the existing TWPSP, although measurements with longer wavelength contain more noise normally.

With Eq. (9), the unwrapped phase phi_3 can be obtained, and the unwrapped phase phi_3 from the existing TWPSP method is shown in Fig. 9(a), while the unwrapped phase phi_3 from the proposed TWPSP method is shown in Fig. 9(b). It is seen that there are a lot of points are incorrectly unwrapped in Fig. 9(a). In contrast, almost all the points are unwrapped correctly in Fig. 9(b) except the region without the object. 


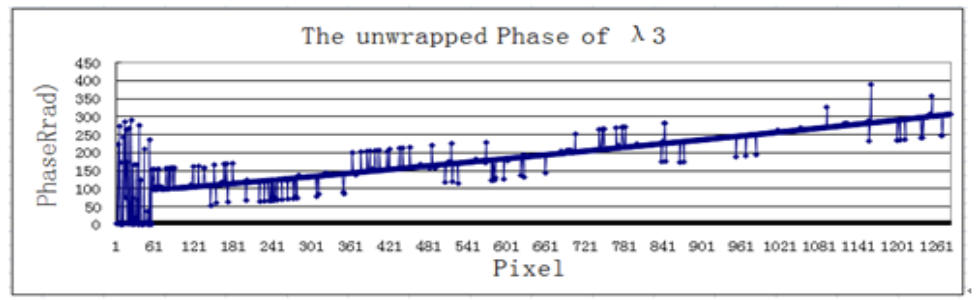

(a)

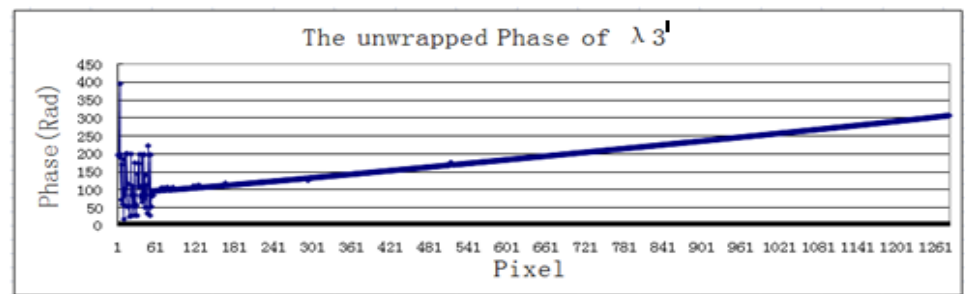

(b)

Fig. 9. (a) The unwrapped phase of the existing TWPSP method with

$\lambda_{3}$. (b) The unwrapped phase of the proposed TWPSP method with $\lambda_{3}{ }^{\prime}$

The results in the above figures clearly show that the noise contained in the unwrapped phases of the proposed method is much less than that of the existing method. In order to quantitatively analyze the noise, 20 points (70-89) are selected, and the RMSEs of the methods are shown in Table 2, which again demonstrate the advantage of the proposed method.

Table 2: RMSE of the two methods

\begin{tabular}{|c|c|c|c|}
\hline \multirow[b]{2}{*}{ ID } & & & unwrapped \\
\hline & $\begin{array}{l}\text { The expected } \\
\text { unwrapped phase } \\
\text { value (Rad) }\end{array}$ & $\begin{array}{l}\text { The unwrapped } \\
\text { phase from the } \\
\text { existing method ( } \mathrm{Rad})\end{array}$ & $\begin{array}{l}\text { phase from the } \\
\text { proposed method } \\
\text { (Rad) }\end{array}$ \\
\hline RMSE & 0 & 33.0999 & 2.9645 \\
\hline
\end{tabular}

In order to test the performance of the proposed method, four different methods are applied for the measurement of the same object in the same environment, 
including the proposed method, the existing TWPSP method, the Gray Code method, and the Multi Wavelength method. The object is a bright hand model placed on a dark chair.

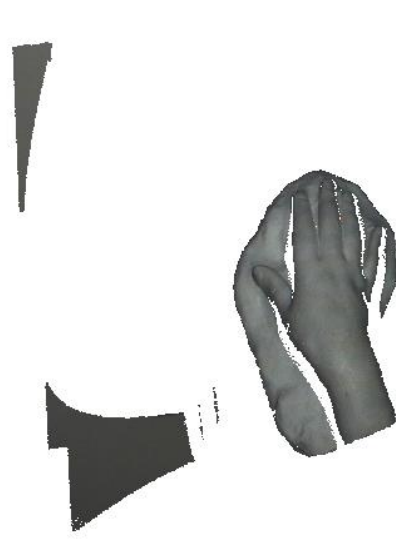

(a)

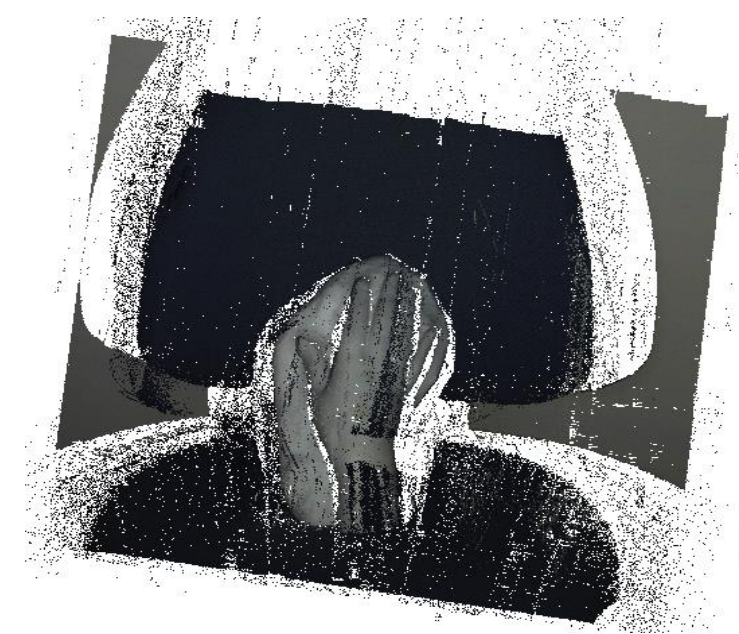

(c)

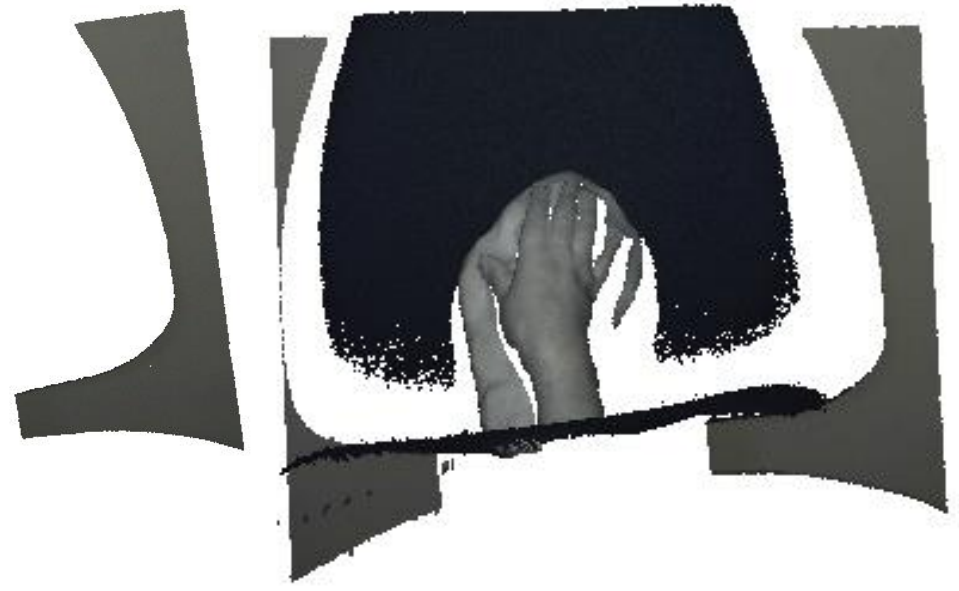

(b)

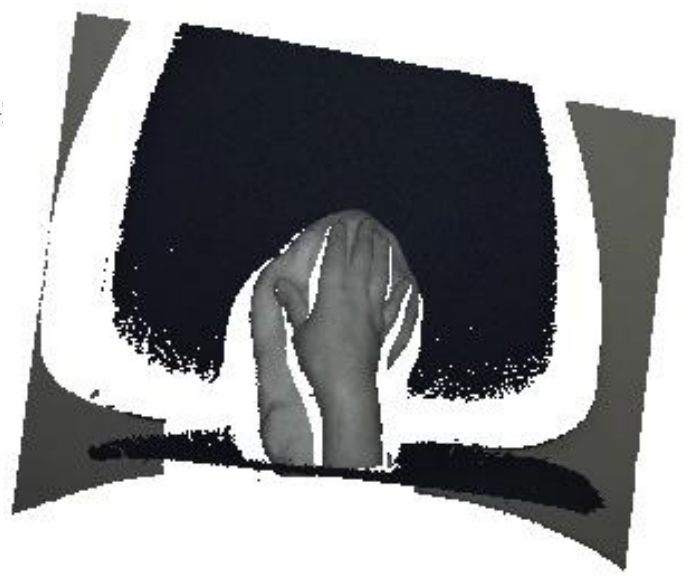

(d)

Fig. 10. Experiment results of the different methods. (a) The 3D reconstruction result from the GC (Gray code) PSP method. (b) The 3D reconstruction result from the MWPSP method. (c) The 3D reconstruction result using the existing TWPSP method. (d) The 3D reconstruction result using the proposed TWPSP method.

The 3D reconstruction result from the GCPSP method and MWPSP method are shown in Fig. 10 (a) and Fig. 10(b) respectively. For GCPSP method, the dark chair 
region is lost, and only bright region remains. For the MWPSP method, the dark region and the bright region are all retrieved clearly. The $3 \mathrm{D}$ reconstruction result using the existing TWPSP method is shown in Fig. 10 (c). There are a lot of noise caused by the incorrectly phase value. The $3 \mathrm{D}$ reconstruction result using the proposed TWPSP method is shown in Fig. $10(\mathrm{~d})$. The 3D reconstruction result is much better, and the dark region is well reconstructed with small noise.

To further examine the performance of the proposed method, we conduct another experiment with two color dolls put on a dark chair. The reconstruction results with different methods are shown in Fig. 11.

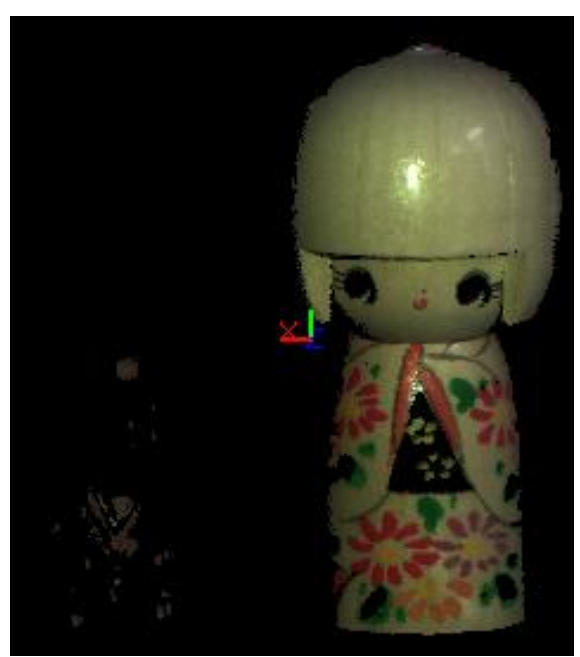

(a)

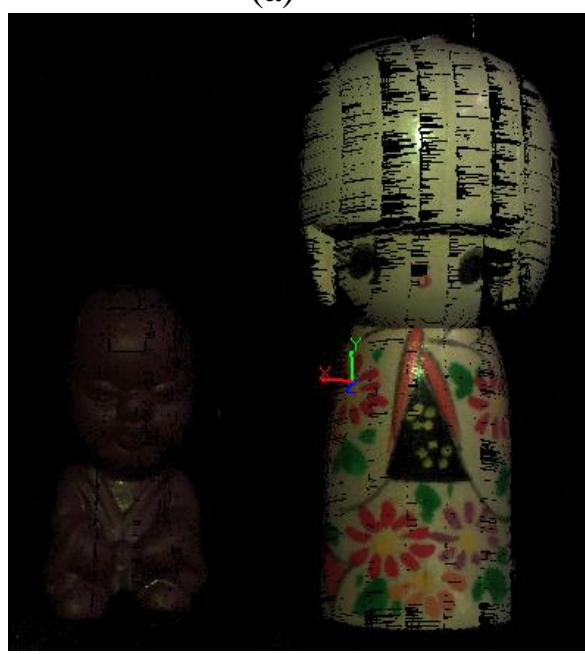

(c)

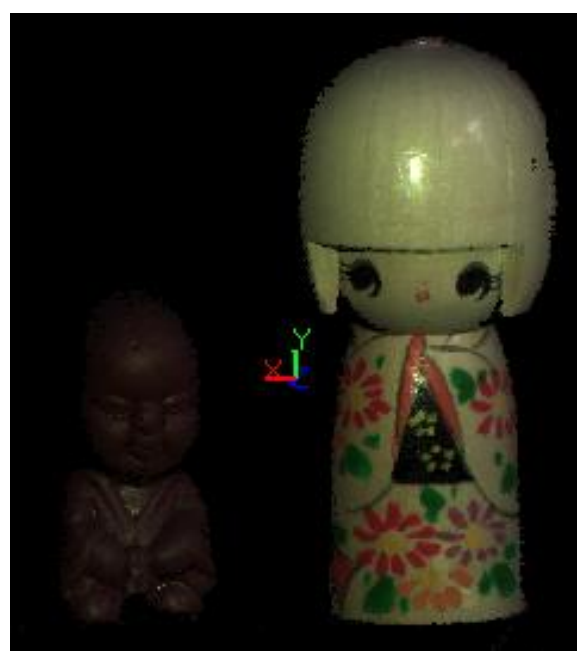

(b)

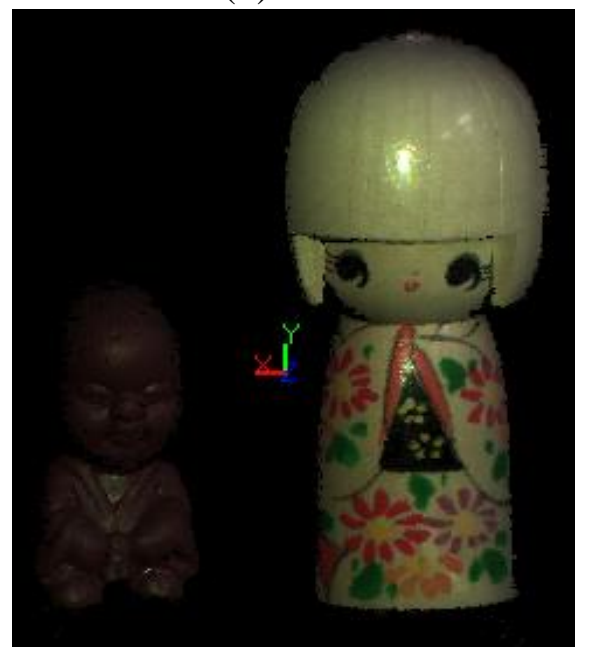

(d) 
Fig. 11. Experiment results of the different methods. (a) The 3D reconstruction result from the GCPSP method. (b) The 3D reconstruction result from the MWPSP method. (c) The 3D reconstruction result using the existing TWPSP method. (d) The 3D reconstruction result using the proposed TWPSP method.

From the figure, we can find that quality of the reconstructed dark doll is different. The dark doll is well reconstructed in the MWPSP method and the proposed TWPSP method. However, the dark doll is lost in the GCPSP method, and the reconstructed dark doll contains a lot of noise in the existing TWPSP method.

From the above two experiments, we can find that the MWPSP method the TWPSP method have similar performance, but the proposed method is faster than the MWPSP method because the proposed one does not need to calculate the equivalent wavelengths and the equivalent phases. The comparisons among the four methods are summarized in Table 3.

Table 3. The comparison between the four methods

\begin{tabular}{|c|c|c|c|c|}
\hline & \multicolumn{1}{|c|}{ The } & \multicolumn{1}{c|}{ The } & \multicolumn{1}{c|}{ The } \\
& proposed & MWisting & MWPS & Gray Code \\
& TWPSP method & TWPSP & method & method \\
\hline Number of & 9 & method & 18 & 9 \\
images captured & & 9 & High & Low \\
\hline Precision & High & Middle & 45 & 15 \\
\hline Processing & 12 & 27 & & \\
speed(in second) & & & & \\
\hline
\end{tabular}




\begin{tabular}{|l|l|l|l|l|}
\hline Robustness & High & Low & High & Low \\
\hline
\end{tabular}

\section{Conclusions}

A new TWPSP method is presented in this paper. In the proposed method, three fringe patterns with proper wavelengths are projected on the object, and the wrapped phase can be obtained with the three proper wavelengths directly, i.e., the calculations of the equivalent wavelengths and their corresponding phase maps are not needed. This leads to the following advantages of the proposed method compared to the existing methods:

1) Low noise: In the proposed method, the wrapped phase can be obtained with the three proper wavelengths directly. Therefore, the noise enhancement problem is alleviated, thereby leading to better performance. It is found that the greater the noise in the environment, the more performance improvement the proposed method can achieve.

2) High speed: The proposed method does not need to calculate the equivalent phase maps, so the speed of the proposed method is higher compared with other methods. Moreover, through experiments, we also found that the reconstruction performance of the proposed method is close to that of the MWPSP method.

\section{Acknowledgments}

This research is supported by the National Natural Science Foundation of China (N0.60808020 and 61078041), the National Science and Technology Support (2014BAH03F01), the Tianjin Research Program of Application Foundation and Advanced Technology (No. 10JCYBJC07200), and the Technology program of Tianjin Municipal Education Commission (20130324). We thank National Natural 
Science Foundation Committee and Tianjin Research Program of Application Foundation and Advanced Technology for the support. This research is also supported by the Tianjin Key Laboratory of Advanced Electrical Engineering and Energy Technology.

\section{References}

[1] K. Chen, J. Xi, Y. Yu, S. Tong, and Q. Guo, “Three-dimensional measurement of object surfaces with complex shape and color distribution based on projection of color fringe patterns,” Appl. Opt. 52(30), 7360-6(2013).

[2] F. Da, and F. Dong, "Windowed Fourier transform profilometry based on improved S-transform," Opt. Lett. 37(17), 3561-3(2012).

[3] J. C. Gutiérrez-García, J. F. Mosiño, A. Martínez, T. A. Gutiérrez-García, E. Vázquez-Domínguez, and J. Arroyo-Cabrales, "Practical eight-frame algorithms for fringe projection profilometry," Opt. Express. 21(1), 903-17(2013).

[4] C. Zuo, Q. Chen, S. Feng, F. Feng, G. Gu, and X. Sui, "Optimized pulse width modulation pattern strategy for three-dimensional profilometry with projector defocusing,” Appl. Opt. 51(19), 4477-92012(2012).

[5] Y. Xiao, Y. Cao, and Y. Wu, "Improved algorithm for phase-to-height mapping in phase measuring profilometry," Appl. Opt. 51(8), 1149-55(2012).

[6] W. Lohry, V. Chen, and S. Zhang, "Absolute three-dimensional shape measurement using coded fringe patterns without phase unwrapping or projector calibration," Opt Express. 22(2):1287-301(2014).

[7] S. Li, W. Chen, and X. Su, "Reliability-guided phase unwrapping in 
wavelet-transform profilometry," Appl. Opt. 47, 3369-99 (2008).

[8] S. J. Feng, Y. Z. Zhang, Q. Chen, C. Zuo, R. B. Li, and G. C. Shen, "General solution for high dynamic range three-dimensional shape measurement using the fringe projection technique,” Opt. Lasers Eng. 59, 56-71(2014).

[9] J. M. Huntley, and H. O. Saldner, "Error-reduction methods for shape measurement by temporal phase unwrapping, " J. Opt. Soc. Am. A. 14, 3188-3196(1997).

[10] D. L. Zheng, and F. P. Da, "Self-correction phase unwrapping method based on Gray-code light,” Opt. Lasers Eng. 50(8), 1130-1139(2012).

[11] Q. C. Zhang, X. Y. Su, L. Q. Xiang, and X. Z. Sun, "3-D shape measurement based on complementary Gray-code light,” Opt. Lasers Eng. 50(4), $574-579(2012)$.

[12] Y. M. Chen, Y. M. He, E. Y. Hu, and H. M. Zhu, "Deformation measurement using dual-frequency projection grating phase-shift profilometry," Acta Mech. Solida Sin. 21(2), 110-115(2008).

[13] C. E. Towers, D. P. Towers, and J. D. C. Jones, "Optimum frequency selection in multi-frequency interferometry," Opt. Lett. 28, 1-3(2003).

[14] C. E. Towers, D. P. Towers, and J. D. C. Jones, “Absolute fringe order calculation using optimised multi-frequency selection in full-field profilometry," Opt. Laser Eng. 43, 788-800(2005).

[15] Y. Li, W. Xiao, and F. Pan, "Multiple wavelength scanning based phase unwrapping method for digital holographic microscopy,” Appl Opt. 
53(5), 979-87(2014)

[16] Y. Ding, J. T. Xi, Y. G. Yu, and J. Chicharo, "Recovering the absolute phase maps of two fringe patterns with selected frequencies," Opt. Lett. 36(13), 2518-2520(2011).

[17] Y. Ding, J. T. Xi, Y. G. Yu, W. Q. Cheng, S. Wang, and J. Chicharo, "Frequency selection in absolute phase maps recovery with two frequency projection fringes," Opt. Express. 20(12),13238-13251( 2012).

[18] J. L. Long, J. T. Xi, M. Zhu, W. Q. Cheng, R. Cheng, Z. W. Li, and Y. S. Shi "Absolute phase map recovery of two fringe patterns with flexible selection of fringe wavelengths," Appl. Opt. 53(9), 1794-1801(2014).

[19] L. M. Song, X. X. Dong, J. T. Xi, Y. G. Yu, and C. K. Yang, "A new phase unwrapping algorithm based on Three Wavelength Phase Shift Profilometry method," Opt. Laser Technol. 45, 319-329 (2013).

[20] L. M. Song, Y. L. Chang, Z. Y. Li, P. Q. Wang, G. X. Xing, and J. T. Xi, "Application of global phase filtering method in multi frequency measurement", Opt. Express. 22(11), 13641-13647(2014). 